\title{
X Congreso lbérico de Agroingeniería

\section{Determinación del consumo de agua en lechuga (Lactuca Sativa L CV Neruda) mediante lisimetría de pesada compacta y sondas de humedad}

\author{
A. Fernández López ${ }^{1}$, M. Soler-Méndez², M.J. Oates², J.M. Molina-Martínez², A. Ruiz- \\ Canales $^{1}$ \\ 1 Departamento de Ingeniería, Universidad Miguel Hernández de Elche, Escuela Politécnica Superior de \\ Orihuela (EPSO-UMH). Crtra. de Beniel, km 3,2. 03312 Orihuela (Alicante), Spain. \\ 2 Grupo de I+D+i de Ingeniería Agromótica y del Mar. UPCT. C/ del Ángel s/n. Ed. ELDI E1.06.30202 \\ Cartagena (Murcia), Spain
}

Resumen: Se desarrollaron los ensayos en una parcela experimental de la Escuela Politécnica Superior de Orihuela (EPSO) de la Universidad Miguel Hernández de Elche (UMH). En la citada parcela se instaló un sistema de riego localizado con cuatro sectores para aplicar cuatro tratamientos diferenciales de fertilización. Se escogió como cultivo de control la lechuga (Lactuca Sativa L Neruda). Se evaluaron las estrategias culturales más adecuadas en el manejo del cultivo en la parcela experimental a partir de los datos periódicos proporcionados por una estación meteorológica próxima y los datos de sensores de humedad del suelo. En uno de los tratamientos se hizo el seguimiento del riego mediante la instalación de un lisímetro de pesada compacto enterrado. La metodología utilizada para la medida simultánea del balance hídrico se ha contrastado y es válida. Es necesario contrastar con más campañas.

Palabras clave: sostenibilidad, ahorro de agua, sensores de bajo coste, programación de riego, horticultura

\section{Introducción}

El uso eficiente del agua de riego disponible es fundamental para conseguir la mejora de la gestión de los recursos hídricos en agricultura [1]. El conocimiento del consumo de agua por los cultivos y el manejo adecuado del riego son factores determinantes. El agua aplicada en exceso o en momentos inadecuados puede provocar efectos no deseados. Un manejo eficiente del agua empleada en riego se fundamenta en la elección del sistema de riego adecuado, la modernización de los regadíos, el ajuste de la dosis de riego a las necesidades reales del cultivo en cada momento y evitar las pérdidas de agua por escorrentía e infiltración fuera del alcance de las raíces. Las mejoras en las técnicas de programación de riego no sólo producen un ahorro del agua, sino que también reduce el riesgo del lavado de nutrientes y minimiza el impacto de la agricultura en las aguas subterráneas. Adicionalmente se asegura el correcto desarrollo del cultivo [2].

La programación del riego se basa principalmente en la determinación de las necesidades hídricas de los cultivos, el conocimiento del estado hídrico de las plantas y la determinación del agua del suelo disponible para el cultivo. El nivel de agua en suelo se puede medir mediante el uso de sensores de humedad instalados de forma apropiada en zonas representativas del cultivo permitiendo observar y estimar la distribución de la humedad y determinar si el riego es excesivo o deficitario [3]. 


\section{CONGRESO IBÉRICO DE AGROINGENIERÍA \\ X CONGRESSO IBÉRICO DE AGROENGENHARIA \\ 3 - 6 septiembre 2019, Huesca - España}

En los últimos años se han desarrollado distintos sistemas que permiten conocer en tiempo real el contenido de humedad del suelo. Suponen una herramienta importante que facilita la toma de decisiones en la programación del riego, tales como determinar la cantidad de agua y el momento de aplicarla. Así, se evitan pérdidas excesivas por percolación profunda o por escorrentía para reducir los impactos ambientales o bien evitar aplicar una cantidad insuficiente y optimizar la producción [4].

A partir de estos sistemas, junto a medios de control y los componentes de riego necesarios (electroválvulas, programadores, etc), se han desarrollado y estudiado métodos de automatización de riego que desencadenan el mismo en función de los niveles de humedad del suelo [5].

Mejorar el manejo del agua en los cultivos, permite conservar agua y aumentar la rentabilidad en las tierras agrícolas. Por lo tanto, el manejo del riego, y con ello su monitorización, constituye una técnica efectiva para obtener cosechas ajustadas, en cuanto a cantidad y calidad, a los requerimientos del mercado.

El objetivo del presente trabajo es el seguimiento del consumo hídrico de cuatro tratamientos diferenciales respecto a las propiedades del suelo de cultivo en lechuga (Lactuca Sativa L cv Neruda). Se emplearon simultáneamente un lisímetro de pesada compacto y sensores de humedad de bajo coste.

\section{Materiales y métodos}

Se desarrollaron los ensayos durante los meses de febrero a marzo de 2017 en una parcela experimental de $300 \mathrm{~m}^{2}$ de la Escuela Politécnica Superior de Orihuela (EPSO) de la Universidad Miguel Hernández de Elche (UMH). En la citada parcela se instaló un sistema de riego localizado con cuatro sectores para aplicar cuatro tratamientos diferenciales respecto a las propiedades del suelo de cultivo. El desarrollo experimental principal de este proyecto fue el estudio del efecto en las propiedades texturales y edáficas del suelo, así como sus efectos en la producción hortícola, de la adición de harina de roca de pórfido, material desarrollado por la empresa Fulsan, S.A., a un suelo agrícola. Se escogió como cultivo de control la lechuga (Lactuca Sativa L Neruda) pues tiene un ciclo corto y se obtienen resultados de una manera relativamente rápida y sencilla. En esta comunicación solamente se muestran los aspectos relacionados con el empleo de sensores de humedad del suelo experimentales y un lisímetro de pesada compacto.

Se evaluaron estrategias de abonado y enmiendas diferenciales en el manejo del cultivo en la parcela experimental a partir de los datos periódicos proporcionados por una estación meteorológica próxima y mediante el uso simultáneo de lisimetría de pesada compacta y sensores de bajo coste en la parcela experimental. Adicionalmente se realizaron análisis periódicos de los principales parámetros de cosecha (peso, producción por superficie, calibre, entre otros).

En el primer tratamiento (T1) se le añadió al suelo al inicio de la campaña una cantidad convenida de harina de roca Fulsan y se aplicó junto con un abonado de fondo inorgánico. Durante las dos campañas de riego de los dos ciclos de cultivo se le aplicó al correspondiente sector (T1) de la plantación de lechuga, además de las correspondientes dosis de riego establecidas, un abonado inorgánico (fertirriego).

Para el segundo tratamiento (T2) se mezclaron harina de roca Fulsan y compost (Bocashi) con el suelo agrícola de la parcela experimental y esta mezcla constituyó un abonado de fondo orgánico. Durante las dos campañas de riego se le aplicó al correspondiente sector (T2) de la plantación de lechuga, además de las correspondientes dosis de riego establecidas, un fertirriego orgánico. El tratamiento T2 no recibió fertirriego inorgánico.

Para el tercer tratamiento (T3) no se añadió ninguna sustancia inicialmente al suelo agrícola. Durante las dos campañas de riego de los dos ciclos de cultivo se le aplicó al correspondiente sector (T3) de la plantación de lechuga, además de las correspondientes dosis de riego establecidas, un abonado inorgánico (fertirriego), como en el tratamiento T1. 


\section{CONGRESO IBÉRICO DE AGROINGENIERÍA \\ X CONGRESSO IBÉRICO DE AGROENGENHARIA \\ 3 - 6 septiembre 2019, Huesca - España}

Adicionalmente se consideró incluir un cuarto tratamiento control (T4) para compararlo con los tratamientos anteriores. A este último tratamiento no se añadió abonado de fondo ni se le añadió fertirriego. Únicamente se le aplicó una dosis de riego similar a los tratamientos anteriores.

Se resumen las cantidades aplicadas en los tratamientos en la tabla 1.

Tabla 1. Tratamientos y cantidades de abono aplicado en el periodo experimental.

\begin{tabular}{ccccc}
\hline & T1 & T2 & T3 & T4 \\
\hline $\begin{array}{c}\text { Harina de pórfido (kgha) } \\
\text { Bocashi (kgha) }\end{array}$ & 210 & 0 & 0 & 0 \\
$\begin{array}{c}\text { Yaara Mila } \\
\begin{array}{c}\text { Complex(kgha) } \\
\text { Durasop Actibion N Plus } \\
\text { (kgha) }\end{array}\end{array}$ & 12,5 & 510 & 0 & 0 \\
\hline
\end{tabular}

El lisímetro empleado es una caja enterrada con cuatro células de carga (ver Fig. 1), que controlan el peso de una bandeja que contiene una porción de suelo y la planta a evaluar (lechuga). Adicionalmente incluye una quinta célula de carga para controlar el peso del agua de drenaje que se pierde por la parte baja de la bandeja. Esta célula de carga se conecta con un depósito de recogida de agua que se vacía periódicamente dependiendo de la cantidad de agua acumulada. Solamente se usó en el tratamiento T2. (Ver resultados y discusión).

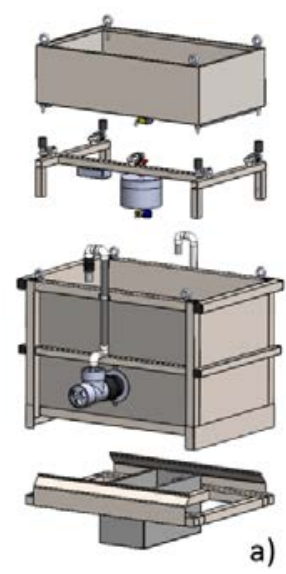

Figura 1. Vistas en perspectiva del lisímetro empleado.

Adicionalmente se usaron sensores capacitivos de bajo coste (Fig. 2). Se trata de sondas capacitivas de dominio de frecuencia. Se conecta a una interfaz electrónica mediante cableado. El microcontrolador utilizado en estos experimentos fue un Mini PCB ArduinoPro, que funciona a $5 \mathrm{v}$ y $16 \mathrm{MHz}$. Las mediciones de temperatura se realizaron utilizando una cámara de temperatura digital impermeable DS18B20, insertada a $3 \mathrm{~cm}$ en el suelo y a $20 \mathrm{~cm}$. Este dispositivo se comunica con el microcontrolador a través del protocolo de cable único. El sistema incluye un cuadro IP56, la sonda capacitiva dominio de la frecuencia, sensor de temperatura DS18B20, amplificador operacional LM358, microcontrolador interfaces y electrónico. Se hizo un registro de cada 5 minutos durante el período experimental. 
X CONGRESO IBÉRICO DE AGROINGENIERÍA

X CONGRESSO IBÉRICO DE AGROENGENHARIA

3 - 6 septiembre 2019, Huesca - España

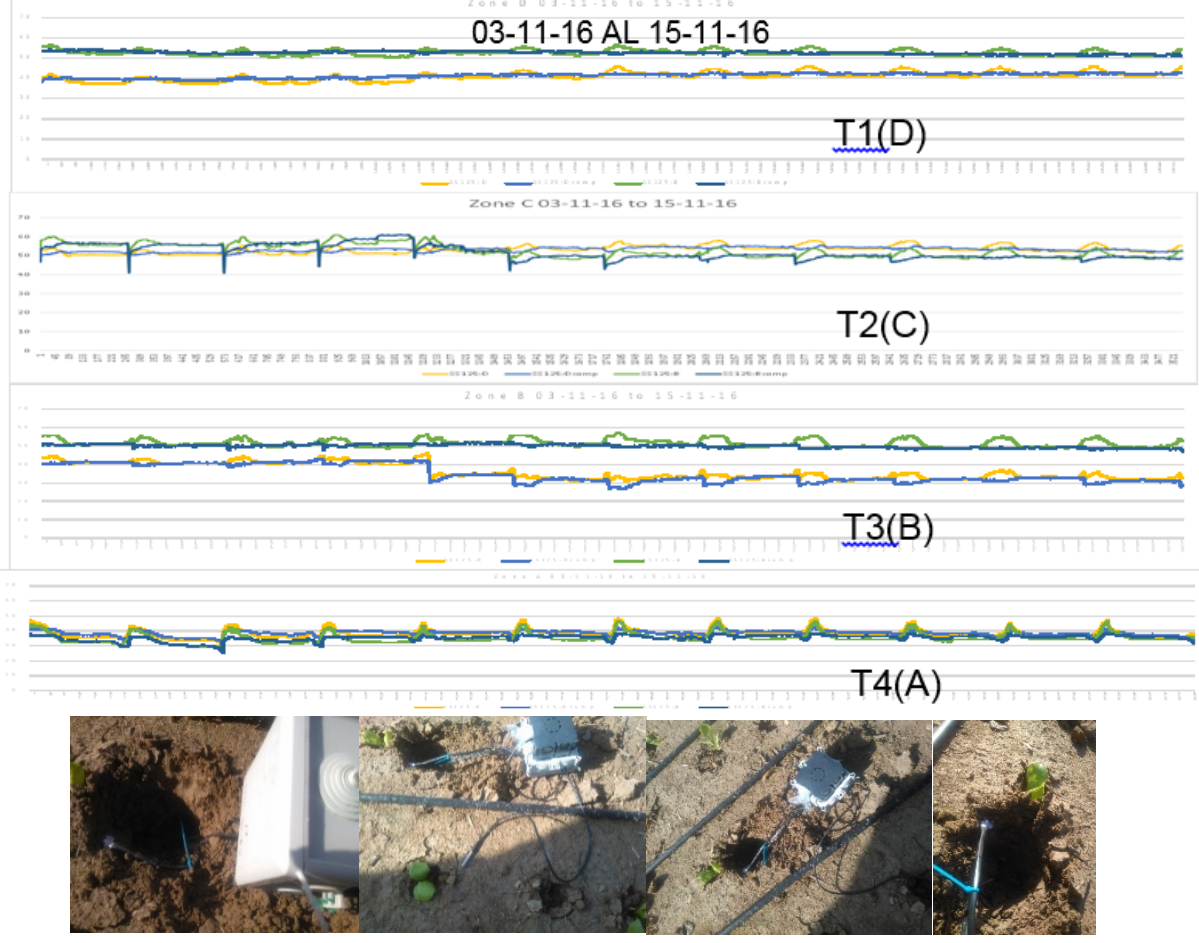

Figura 2. Resultados obtenidos con los sensores de humedad del suelo de bajo coste en los 4 tratamientos y aspectos de su instalación.

La localización del lisímetro y las sondas de humedad del suelo, así como los tensiómetros (watermark) se muestran en la figura 3.
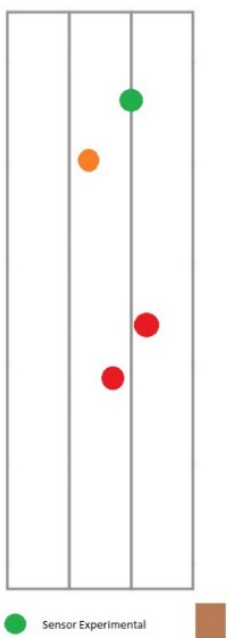
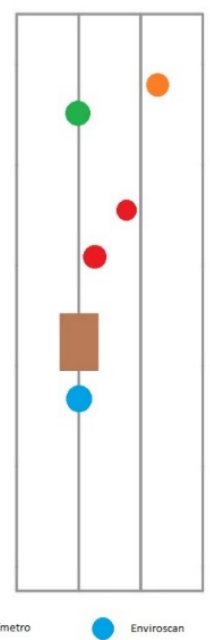
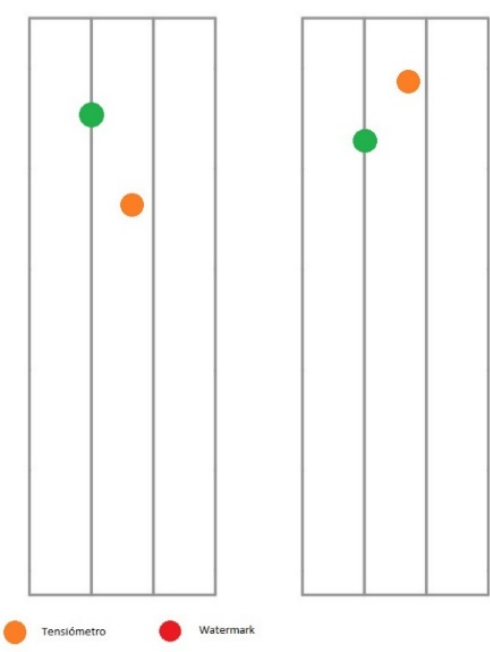

Figura 3. Localización en parcela de los distintos dispositivos: lisímetro, sensor capacitivo de humedad del suelo experimental, sensor capacitivo de humedad del suelo comercial (Enviroscan), tensiómetro (watermark). Se incluyen los 4 bloques empleados con los 4 tratamientos.

\section{Resultados y discusión}

Se muestra en la figura 4 la evolución del peso del lisímetro en un período temporal específico del experimento y se comparó con la cantidad de agua aplicada. 


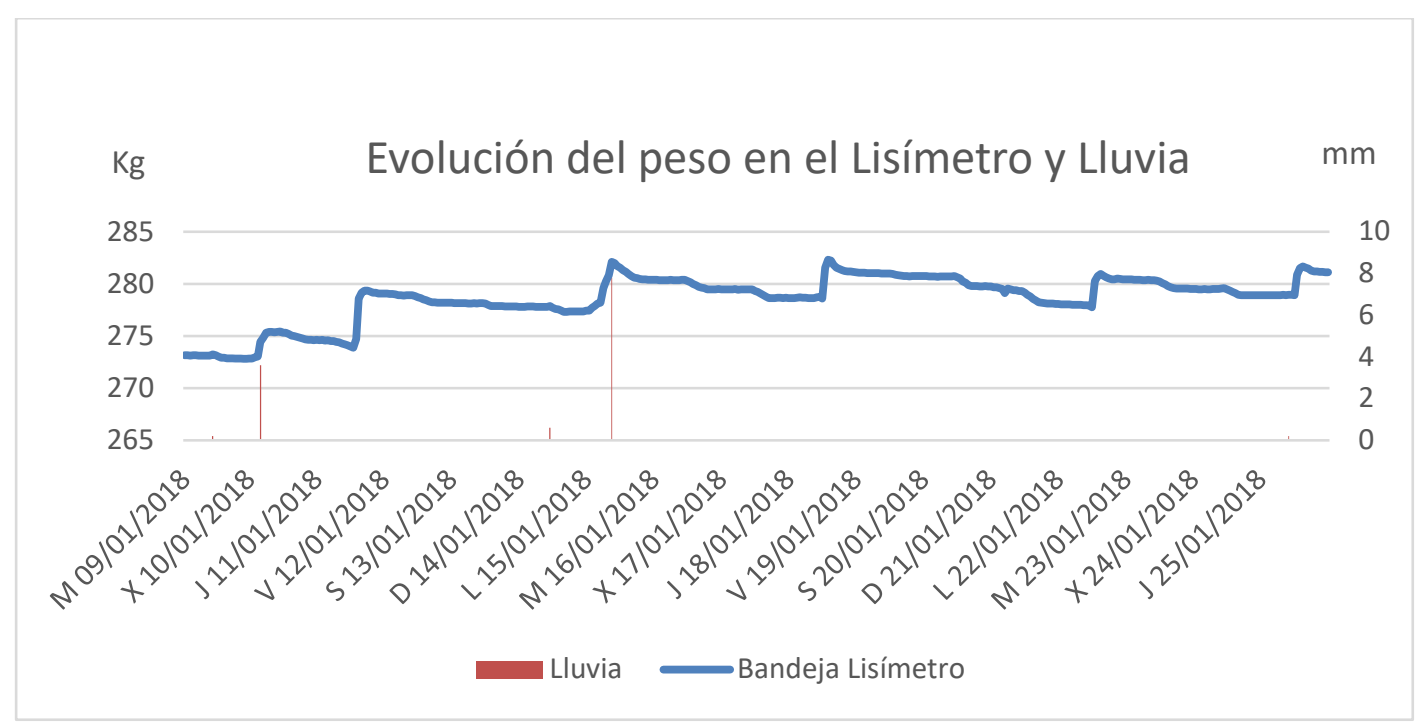

Figura 4. Algunos resultados obtenidos con el lisímetro de pesada con la evolución del peso de la bandeja.

Igualmente se muestra el resultado de las medidas de humedad del suelo de los sensores de humedad (Fig. 2). Se ve que hay una cierta correlación de medidas.

Para el seguimiento del patrón de humedad del cultivo en la zona radicular se instalaron cuatro sensores de humedad de bajo coste (uno por cada tratamiento). Se trata de sensores capacitivos dispuestos a 2 y $10 \mathrm{~cm}$ para controlar la humedad en continuo a lo largo del desarrollo del cultivo de la lechuga. Estos dispositivos incluyen sensores de temperatura del suelo. El valor de la temperatura del suelo es necesario para el calibrado la medida final de la humedad del suelo. Las medidas se registraron en un datalogger y se grabaron en un minidisco. Según se muestra en la Fig. 2, se registraron los episodios de riego a lo largo del período de cultivo y la variación de humedad en las dos profundidades citadas. Estas medidas se contrastaron con los aportes de agua medidos con contador. Adicionalmente permitieron establecer las cantidades de agua drenada para determinar el balance hídrico. Del mismo modo, se contrastaron las medidas estimadas del drenaje con las obtenidas mediante lisimetría de pesada. Aunque puntualmente dieron resultados similares, esta técnica debe contrastarse con más medidas en campo.

Las cantidades de agua registrada en el experimento se muestran en la tabla 2. Se concluye con que la adición de harina de pórfido tiene un efecto significativo en la retención de agua del suelo.

Tabla 2. Consumos de agua para el periodo experimental.

\begin{tabular}{ccccc}
\hline & T1 & T2 & T3 & T4 \\
\hline Riego (mm) & 91,2 & 96,3 & 102,8 & 109,0 \\
Lluvia (mm) & 63,4 & 63,4 & 63,4 & 63,4 \\
$\begin{array}{c}\text { Agua total } \\
(\mathrm{mm})\end{array}$ & 154,6 & 159,7 & 166,2 & 172,4 \\
$\begin{array}{c}\text { Agua total } \\
\text { utilizada (mm) }\end{array}$ & 92,6 & 99,0 & 99,6 & 103,2 \\
$\begin{array}{c}\text { Agua total } \\
\text { drenada }(\mathrm{mm})\end{array}$ & 61,3 & 60,0 & 66,7 & 69,1 \\
\hline
\end{tabular}




\section{CONGRESO IBÉRICO DE AGROINGENIERÍA \\ X CONGRESSO IBÉRICO DE AGROENGENHARIA \\ 3 - 6 septiembre 2019, Huesca - España}

\section{Conclusiones}

La metodología utilizada para la medida simultánea del balance hídrico se ha contrastado y es válida. Es necesario contrastar con más campañas.

La influencia de la enmienda en el crecimiento vegetativo no ha sido validada. No obstante, según las sondas de humedad del suelo, hay una diferencia en cuanto al movimiento de agua en el suelo en el T1 respecto a los demás tratamientos. Es posible que la harina de pórfido en combinación con el abono inorgánico mejore el movimiento del agua en el suelo y mejore el perfil de humedad.

\section{Agradecimientos}

Los autores del artículo expresan su agradecimiento a la empresa FULSAN, S.A. por su apoyo económico. Igualmente se muestran agradecidos a la empresa TELENATURA EBT, S.L. y Viveros Simón Cases, S.L. por su apoyo tecnológico y agronómico.

\section{Referencias}

1. Kayatz, B.; Harris, F.; Hillier, J.; Adhya, T.; Dalin, C.; Nayak, D.; Green, R. F.; Smith, P.; Dangour, A. D. "More crop per drop": Exploring India's cereal water use since 2005. The Science of the total environment, 2019, 673 (207-217).

2. Kambou, D ; Degre, A., Xanthoulis, D., Ouattara, K., Destain, J.P., Defoy, S., De L'escaille, D. Evaluation and Proposals for Improving Irrigation Performance Around Small Reservoirs in Burkina Faso. Journal of irrigation and drainage engineering, 2019, 145, 6, 05019004.

3. Welde, K., Gebremariam, H.L., Kahsay, K.D. Optimizing irrigation water levels to improve yield and water use efficiency of vegetables: case study of tomato. Sustainable water resources management, 2019, 5, 2 (737-742).

4. Hajdu, I., Yule, I., Bretherton, M., Singh, R., Hedley, C. Field performance assessment and calibration of multi-depth AquaCheck capacitance-based soil moisture probes under permanent pasture for hill country soils. Agricultural water management, 2019, 217 (332-345).

5. Zheng, Z.L., Wang, Z.J., Zhao, J.S., Zheng, H. Constrained Model Predictive Control Algorithm for Cascaded Irrigation Canals. Journal of irrigation and drainage engineering, 2019, 145, 6, 04019009. 